\title{
Novel leptin receptor signaling mutants identify location and sex-dependent modulation of bone density, adiposity, and growth
}

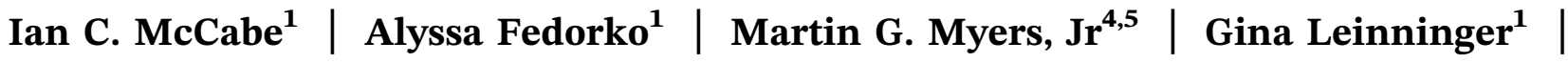 \\ Erica Scheller ${ }^{6}$ Laura R. McCabe M,2,3 $^{1,0}$
}

${ }^{1}$ Department of Physiology, Michigan State University, East Lansing, Michigan

${ }^{2}$ Department of Radiology, Michigan State University, East Lansing, Michigan

${ }^{3}$ Biomedical Imaging Research Center, Michigan State University, East Lansing, Michigan

${ }^{4}$ Department of Molecular and Integrative Physiology, Division of Metabolism, Endocrinology and Diabetes, University of Michigan, Ann Arbor, Michigan

${ }^{5}$ Department of Internal Medicine, University of Michigan, Ann Arbor, Michigan

${ }^{6}$ Department of Medicine, Division of Bone and Mineral Diseases, Washington University, Saint Louis, Missouri

\section{Correspondence}

Laura R. McCabe, Department of Physiology, Michigan State University, 2201 Biomedical Physical Science Building East Lansing, MI 48824.

Email: mccabel@msu.edu

\section{Funding information}

National Institute of Diabetes and Digestive and Kidney Diseases, Grant/ Award Numbers: RO1 DK101050, DK056731; National Center for Complementary and Integrative Health, Grant/Award Number: RO1 AT007695; National Institute of Dental and Craniofacial Research, Grant/Award Number: R00 DE024178

\begin{abstract}
Leptin, a hormone primarily produced by adipocytes, contributes to the regulation of bone health by modulating bone density, growth and adiposity. Upon leptin binding, multiple sites of the long form of the leptin receptor (LepRb) are phosphorylated to trigger activation of downstream signaling pathways. To address the role of LepRb-signaling pathways in bone health, we compared the effects of three LepRb mutations on bone density, adiposity, and growth in male and female mice. The $\Delta 65$ mutation, which lacks the known tyrosine phosphorylation sites, caused obesity and the most dramatic bone phenotype marked by excessive bone adiposity, osteoporosis, and decreased growth, consistent with the phenotype of $\mathrm{db} / \mathrm{db}$ and ob/ob mice that fully lack leptin receptor signaling. Mutation of LepRb $\mathrm{Tyr}_{1138}$, which results in an inability to recruit and phosphorylate signal transducer and activator of transcription 3, also caused obesity, but bone loss and adiposity were more dominant in male mice and no growth defect was observed. In contrast, mutation of LepRb Tyr $_{985}$, which blocks SHP2/SOCS3 recruitment to LepRb and contributes to leptin hypersensitivity, promoted increased femur bone density only in male mice, while marrow adiposity and bone growth were not affected. Additional analyses of vertebral trabecular bone volume indicate that only the $\operatorname{Tyr}_{1138}$ mutant mice exhibit bone loss in vertebrae. Together, our findings suggest that the phosphorylation status of specific sites of the LepRb contribute to the sex- and location-dependent bone responses to leptin. Unraveling the mechanisms by which leptin responses are sex- and locationdependent can contribute to the development of uniquely targeted osteoporosis therapies.
\end{abstract}

\section{K E Y W O R D S}

adipocytes, bone density, bone volume, female, leptin receptor, male, marrow adiposity, obesity, osteoporosis 


\section{INTRODUCTION}

Leptin is a $16 \mathrm{kDa}$ hormone/adipokine produced primarily by adipocytes and is involved in the regulation of body mass and bone density. ${ }^{1-7}$ Serum leptin levels are positively linked with body fat stores. In the brain, leptin signals through neurons expressing the long form of the leptin receptor (LepRb) to communicate the status of the body's energy reserves. ${ }^{8,9}$ When adipose tissue is abundant, increased serum leptin levels lead to increased energy expenditure, decreased food intake, and weight loss. Conversely, the absence of leptin causes a reduction in energy expenditure, increased food intake and weight gain. Leptin also directly regulates peripheral cell function by binding to leptin receptors (LRs) expressed on a variety of cell types including mesenchymal stem/ stromal cells (MSCs), which can mature into osteoblasts or adipocytes. ${ }^{10-14}$

The effects of leptin on bone are complex. Leptin can stimulate or inhibit bone formation depending upon bone location and whether leptin is acting directly via receptors on MSCs and/or osteoblasts ${ }^{10,13,14}$ or indirectly through LepRb-expressing hypothalamic neurons that can polysynaptically regulate bone. ${ }^{1,14,15}$ Mice that are completely leptin-deficient (ob/ob mice) have age-dependent, site-specific phenotypes including lower femoral bone mineral content, cortical thickness, bone mineral density (BMD), trabecular bone volume, and decreased bone length. ${ }^{1,2,16-18}$ Similarly, leptin receptor deficiency (db/db mice) causes decreased tibial trabecular bone volume, bone length, and cortical thickness. ${ }^{18,19}$ Absence of leptin or LepRb in mice also increases bone marrow adiposity. ${ }^{1,17,20}$ The receptor-mediated mechanisms underlying these skeletal and marrow adiposity phenotypes remain unknown.

The LR belongs to the class I cytokine receptor superfamily and is encoded by a single gene (Lepr). Several alternatively spliced LR isoforms exist and can be divided into three classes: secreted, long, and short. ${ }^{21}$ Leptin initiates its action through binding to the single long form of the leptin receptor (LepRb), which leads to the autophosphorylation and activation of the LepRbassociated Jak2 protein. Jak2, in turn, phosphorylates three critical LepRb tyrosine residues $\left(\mathrm{Tyr}_{985}, \mathrm{Tyr}_{1077}\right.$, and $\mathrm{Tyr}_{1138}$ ) located in an intracellular domain that is composed of $\sim 300$ residues. Each of the phosphorylation sites can recruit specific Src homology 2 (SH2) domaincontaining proteins. Specifically, phosphorylation of the first site, $\mathrm{Tyr}_{985}$, recruits Src homology phosphatase-2 (SHP-2) as well as a suppressor of cytokine signaling 3 (SOCS3), which leads to an attenuation of LepRb signaling. ${ }^{21}$ Phosphorylation of the second site, $\mathrm{Tyr}_{1077}$, recruits the signal transducer, latent transcription factor, and signal transducer and activator of transcription 5 (STAT5). ${ }^{22}$ Phosphorylation of $\mathrm{Tyr}_{1138}$ results in the recruitment of STAT3. ${ }^{23,24}$ Thus, each phosphorylation site activates a unique signaling cascade to mediate distinct aspects of leptin action.

Genetically modified mice containing mutations in the LepRb can be used to determine the role of each of the three key phosphorylation sites on mouse physiology. Mice were generated by replacing the LepRb-specific exon $18 \mathrm{~b}$ of Lepr with a mutant exon $18 \mathrm{~b}$. This genetargeting strategy expresses mutant LepRb molecules from the genomic context of endogenous LepRb so that expression patterns and levels of mutant LepRb mirror those of wild-type (WT) LepRb. ${ }^{25}$ Mice expressing a mutation in $\mathrm{Tyr}_{985}$ (termed LL mice) have abrogated phosphorylation of the site and blocked SHP2/SOCS3 recruitment. LL mice are lean and display leptin hypersensitivity. Conversely, mice expressing a mutation in $\mathrm{Tyr}_{1138}$ (termed SS mice), are unable to recruit STAT3 to LepRb and display an obese and hyperphagic phenotype marked by decreased energy expenditure with increased growth. ${ }^{25}$ Finally, $\Delta 65$ animals lack LepRb signaling and are similar to $\mathrm{db} / \mathrm{db}$ mice. ${ }^{26}$ To understand the role of LepRb and its key phosphorylation sites on bone phenotypes, we carried out an exploratory study examining male and female skeletal parameters (trabecular and cortical; femur and vertebral) in the three LepRb mutant mouse lines and compared our findings to the corresponding WT littermates for each mutant line. Our findings support the complexity of LepRb signaling in the regulation of skeletal health.

\section{MATERIALS AND METHODS}

\subsection{LepRb mutant mouse models}

This study examined LepRb $\Delta 65$, LL, and SS mice on the C57/B16 background, as previously described. ${ }^{22,25,26}$ Heterozygous mice were intercrossed to generate mice homozygous for each LepRb variant and littermate controls used for subsequent studies, which were identified by genotyping between 2 and 4 weeks of age. Study mice were group housed until 8 to 9 weeks of age, then they were anesthetized with a lethal dose of pentobarbital and transcardially perfused with $10 \%$ neutral buffered formalin. Bones were removed and postfixed in formalin for 24 -hours before storage in $70 \%$ ethanol. All mice were bred and housed at the University of Michigan and maintained in a 12-hour light/dark cycle with ad libitum access to food and water. All procedures were approved by the University of Michigan University Committee on the Use and Care of Animals in accordance with Association 
for Assessment and Accreditation of Laboratory Animal Care and National Institutes of Health guidelines.

\section{2 | Bone density measurement}

Femurs and vertebrae (lumbar 3-4) were fixed in $10 \%$ formalin and imaged using a GE Explore Locus microcomputed tomography $(\mu \mathrm{CT})$ system at a voxel of $20 \mu \mathrm{m}$ obtained from 720 views. The beam angle increment was 0.5 and beam strength was set at 80 peak $\mathrm{kV}$ and $450 \mu \mathrm{A}$. Each run consisted of control and mutant mouse bones and a calibration phantom to standardize gray scale values and maintain consistency. Bone measurements were blinded. Maximum vertebral height was determined using GE Healthcare Microview Software (GE Healthcare, London, ON, Canada) and was consistent with previous reports. ${ }^{27}$ Maximum femur length was determined as the distance between the most proximal region of the trochanter to the most distal region of the medial condyle. Distal femur trabecular bone analyses were performed in the metaphyseal region defined at $1 \%$ of the total length $(\sim 0.17 \mathrm{~mm})$ proximal to the growth plate extending $2 \mathrm{~mm}$ toward the diaphysis excluding the outer cortical bone. Trabecular bone volume fraction (BV/TV) was computed by GE Healthcare MicroView software application using a threshold of 700. Cortical bone measurements were determined with a $2-\mathrm{mm}^{3}$ region of interest in the mid-diaphysis.

\section{3 | Leptin serum measurements}

Leptin concentration in female mouse serum was determined by enzyme-linked immunosorbent assay using the Mouse/Rat Leptin Quantikine Kit (R\&D Systems, Minneapolis, MN).

\subsection{Adipocyte counts}

Fixed bones were processed on an automated Thermo Electron Excesior tissue processor for dehydration, clearing, and infiltration using a routine overnight processing schedule. Samples were then embedded in Surgipathembedding paraffin on a Sakura Tissue Tek II-Embedding Center (Sakura Finetek USA, Inc, Torrance, CA). Paraffin blocks were sectioned at $5 \mu \mathrm{m}$ on a Reichert Jung 2030 rotary microtome (Leica Biosystems, Buffalo Grove, IL) and were haemotoxylin and eosin stained. Femur sections were examined by microscopy at $\times 4$ optical zoom and digital images obtained. Images were examined blind to the section's condition. The marrow area starting at $170 \mu \mathrm{m}$ from the growth plate and extending $2000 \mu \mathrm{m}$ toward the diaphysis was measured, by outlining the region and quantifying the area using ImagePro Software
(Media Cybernetics, Rockville, MD). Adipocytes greater than $30 \mu \mathrm{m}$ in diameter were counted and expressed relative to the total marrow area. Analyses were done blinded to conditions.

\subsection{Statistical analyses}

All measurements are presented as the mean \pm SE. All groups contained at least five mice, except for the $\Delta 65$ male mouse group which has three mice. The Student two-tailed $t$ tests were used to determine significance by comparing each genotype to its own littermate and sexmatched controls. Outliers were identified by ROUT and removed (only three identified). Statistical analyses used Student $t$ tests that compared littermate WT to mutant mouse values for each sex. Analyses were performed using the GraphPad Prism Software version 6 (GraphPad, San Diego, CA). A $P \leq 0.05$ was considered significant.

\section{3 | RESULTS}

To determine the role of LepRb and its key phosphorylation sites on the male and female mouse skeleton, we examined 8- to 9-week-old mice that had one of the three LepRb mutations and compared findings to their corresponding sex- and age-matched littermates. We first assessed body mass (Figure 1), which is an important parameter that influences bone phenotype. Consistent with previous studies in $\mathrm{db} / \mathrm{db}$ mice ${ }^{9}$ which lack LepRb signaling, both male and female $\Delta 65$ mice had significantly greater body mass compared with their corresponding littermates, by $49 \%$ and $72 \%$ respectively. An even greater increase was seen in both the male and female SS mutant mice, which displayed significant increases in body mass that were 57\% and $90 \%$ (respectively) greater than their corresponding littermate counterparts. In contrast, the LL mutant mice weighed less. ${ }^{22}$ While the LL mutant males were on average only $4 \%$ less in weight, the female LL mutant mice exhibited a significant $27 \%$ decrease in body weight. This is consistent with the Tyr985 mutation promoting leanness specifically in female mice. ${ }^{9,22}$ Consistent with changes in adiposity and body mass, serum leptin was significantly elevated in SS $(148 \pm 19 \mathrm{ng} / \mathrm{mL})$ and decreased in LL $(1.7 \pm 0.45 \mathrm{ng} / \mathrm{mL})$ compared with corresponding control female mice $(2.8 \pm 0.76$ and $2.76 \pm 0.48)$.

Next, we examined femur bone length to determine if the mutations had any impact on overall bone growth (Figure 2A-C). Both the male and female $\Delta 65$ mice displayed reduced femoral growth compared wtth littermate controls, by $9 \%$ and $12 \%$, respectively. However, no differences were detected for the other genetically 
modified mice, suggesting that signals beyond $\mathrm{Tyr}_{1138}$ induced Stat3 and $\mathrm{Tyr}_{985}$ recruited SHP2/SOCS3 mediate the control of bone length by leptin. We also examined vertebral bone height (L3-L4) but in contrast to another
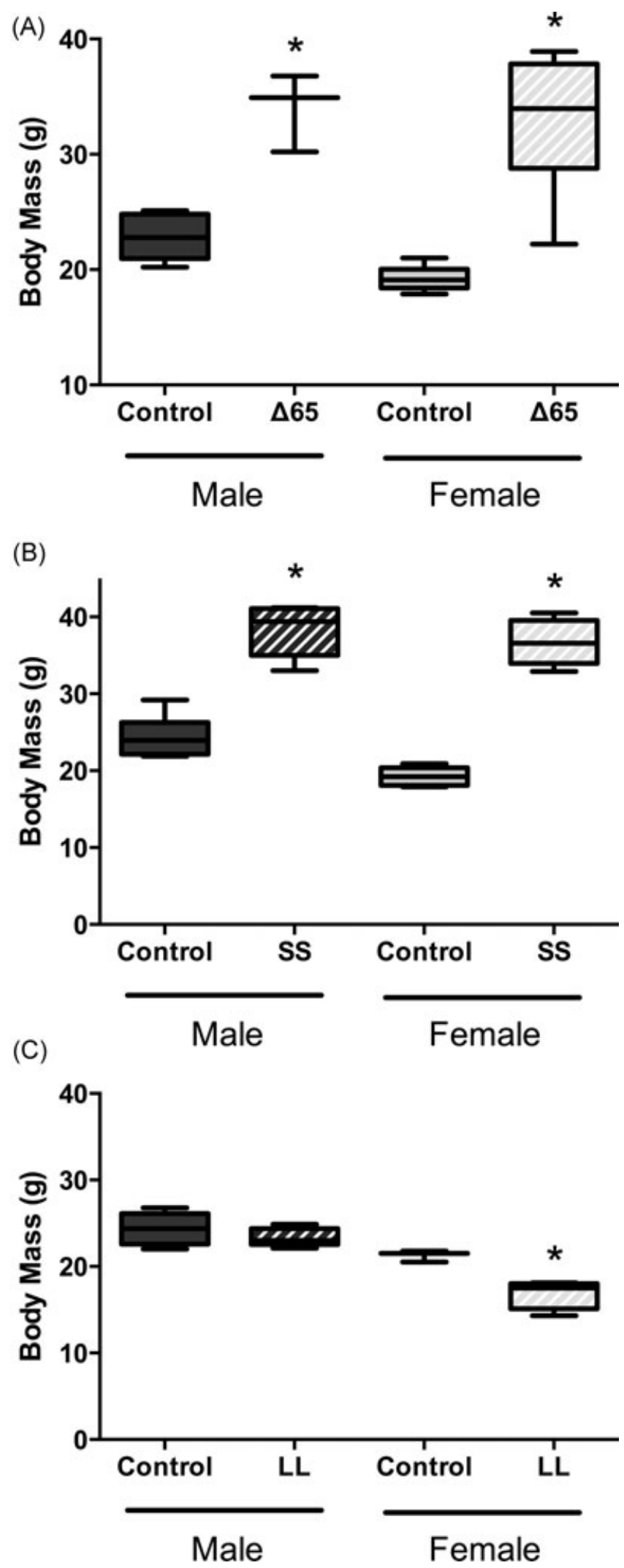

report ${ }^{28}$ we did not observe differences in vertebral height between any of the conditions (data not shown).

To determine if the LepRb mutations affected bone architecture, distal femur trabecular/cancellous bone was examined by microcomputed tomography. Analyses of BV/TV did not differ between conditions except for male LL mutant mice which displayed higher bone volume and greater trabecular thickness compared with controls (Table 1). Male and female $\Delta 65$ mice trended toward a $26 \%$ and $50 \%$ reduction in trabecular BV/TV. By contrast, SS mice, which were of similar body mass, had a trending, though nonsignificant $14 \%$ decrease and $19 \%$ increase in trabecular bone volume fraction. Bone volume was further analyzed relative to body weight since the mice displayed broad differences in body weight (as shown in Figure 1). When corrected for body weight, both male and female $\Delta 65$ mice had significantly reduced femur trabecular bone volume when compared with their corresponding control littermates, by $51 \%$ and $74 \%$ respectively, consistent with previous reports. ${ }^{1,29-31}$ The SS mutant mice showed a significant 53\% decrease for male $\mathrm{BV} / \mathrm{TV}$, and a nonsignificant $43 \%$ decrease for females $(P=0.08)$. In contrast, the LL mutant mice increased bone volume with average significant increase of $52 \%$ for males and a nonsignificant $20 \%$ increase for females, relative to littermate controls.

Examination of vertebral BV/TV, without correction to body weight, show an increase in BV/TV caused by the $\Delta 65$ mutation in male (significant) and female (trend) mice (Table 2). The $\Delta 65$ mutation also caused a significant reduction in vertebral trabecular spacing in both males and females. The SS mutation did not cause any notable effects on vertebral trabecular parameters while the LL mutation induced a significant decrease in trabecular BV/TV in females only. Given the significant differences in body weight between the mouse groups, we also analyzed the BV/TV data relative to body weight and found that there was no longer a difference in BV/TV in the $\Delta 65$ and LL mutant mice compared with their corresponding controls. However, the SS mutant mice exhibited a $22 \%$ decrease in males and a significant $42 \%$ decrease in females (Figure 3E), suggesting that the SS mutant could identify a unique signaling pathway that regulates vertebral bone density (Figure 4).

FI G URE 1 LepRb mutations $\Delta 65$ and SS increase male and female mouse body weight, while the LL mutation causes weight loss in female mice. Body weights were obtained from male and female 8- to 9-week-old mice expressing LepRb mutants: $\Delta 65$, SS $\left(\mathrm{Tyr}_{1138}\right)$, or LL $\left(\mathrm{Tyr}_{985}\right)$. Mutant mouse data are graphed with data obtained from corresponding littermate control mice. Values represent averages \pm SE, $n=3$ to 7 per group, ${ }^{*} P<0.05$ 
Cortical bone parameters were also examined and revealed differences that were sex- and mutation-specific (Table 3). Male LL and SS mutant mice did not show significant cortical differences, however, the $\Delta 65$ muta-

(A)

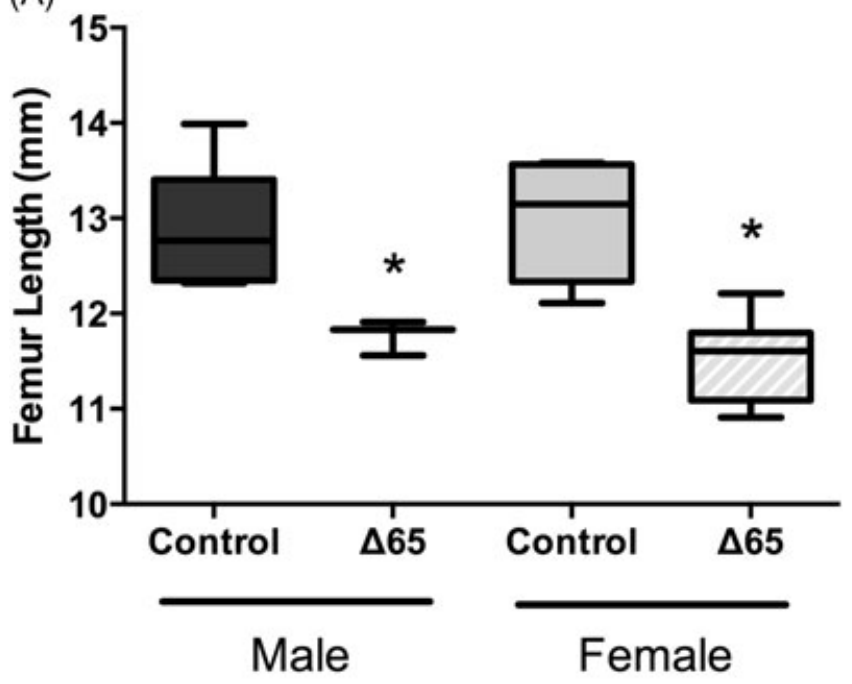

(B)

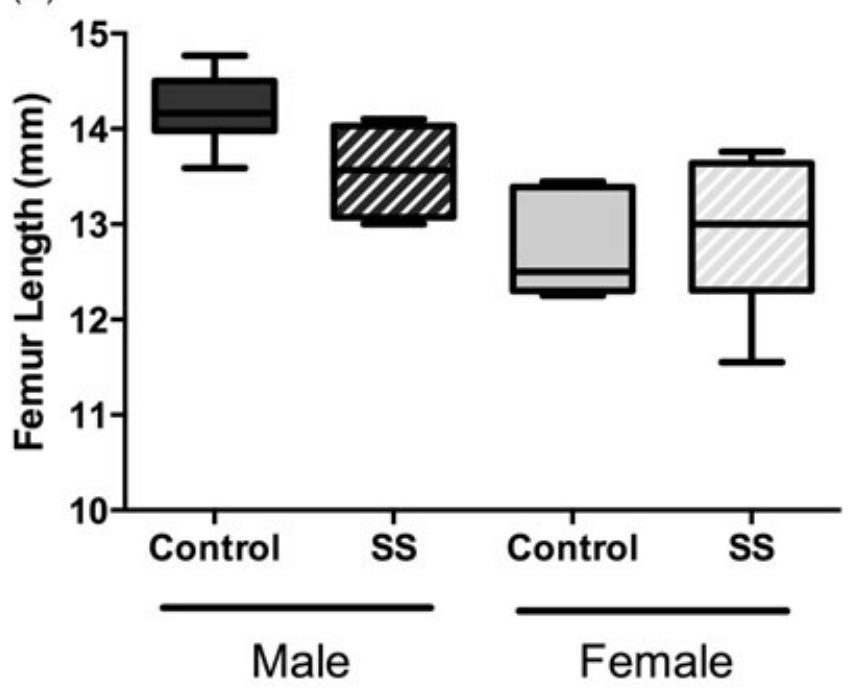

(C)

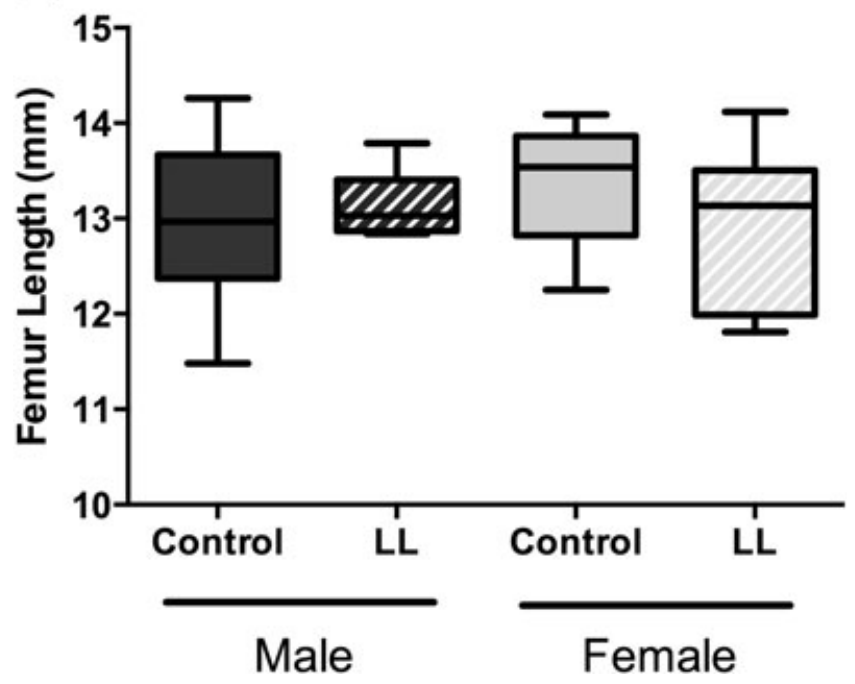

tion displayed less cortical thickness and cortical area compared with WT control mice. Female LepRb mutant mice, on the other hand, displayed several significant differences. Female $\Delta 65$ mice had significantly reduced cortical bone density. Female SS mutant mice had greater inner and outer cortical bone perimeter and marrow area as well as lower BMD compared with WT controls. Regarding the LL mice, females had a smaller cortical inner perimeter than controls, but this did not result in greater mean thickness or cortical area (Table 2).

Previously, our lab and others ${ }^{10-12,14,32}$ demonstrated that leptin can influence marrow adiposity. Therefore, we further examined the effect of the LR mutations on marrow adiposity. Adiposity was markedly increased in the male $\Delta 65$ mice by a significant 10.3-fold (to an average of 72 adipocytes $/ \mathrm{mm}^{2}$ ). While adiposity was also significantly increased in the SS male mutants, the increase was modest, 3.5 -fold (to an average between 3 and 4 adipocytes $/ \mathrm{mm}^{2}$ ), compared with the effect of $\Delta 65$ on marrow fat. The female $\Delta 65$ mice exhibited an average increase of 2.7 -fold (to 51 adipocytes $/ \mathrm{mm}^{2}$ ) and the SS female mice displayed a 1.5-fold average increase (to $\sim 5$ adipocytes $/ \mathrm{mm}^{2}$ ) compared with littermate controls, though these results did not reach statistical significance. The LL mutation did not impact marrow adiposity in either the male or female mice.

\section{DISCUSSION}

Past studies demonstrate a role for leptin in the regulation of bone density and adiposity. Using mice expressing different LepRb-signaling mutations, we identified distinct roles for LR signaling pathways in the regulation of bone density, adiposity, or growth. By far the strongest phenotype we obtained was from the $\Delta 65$ mice, which lack LR signaling via characterized intracellular tyrosine residues. This mutation made both male and female mice obese while also decreasing bone density and growth and increasing marrow adiposity in mouse femurs. While the male mutant mouse number was underpowered $(n=3)$, our findings are consistent with reports on bone phenotypes caused by either leptin deficiency as seen in ob/ob mice or in $\mathrm{db} / \mathrm{db}$ mice that lack LepRb. ${ }^{1,29-31}$ The SS mutation of LepRb $\mathrm{Tyr}_{1138}$, which prevents LepRb-

FI G U RE 2 Only LepRb mutation $\Delta 65$ effected femur length/ growth. Femur lengths were measured for male and female 8 to 9-week-old mice expressing LepRb containing mutations $\Delta 65$, SS, or LL. Mutant mouse data are graphed and analyzed relative to data obtained from corresponding littermate control mice. Values represent averages \pm SE. $\Delta 65$ mice, $n \geq 3$ per group; SS and LL mice, $n=6$ to 7 per group; ${ }^{*} P<0.05$ 
TABLE 1 Femoral trabecular bone parameters

\begin{tabular}{|c|c|c|c|c|}
\hline & \multicolumn{2}{|l|}{ Male } & \multicolumn{2}{|l|}{ Female } \\
\hline & Control & Mutant & Control & Mutant \\
\hline $\mathrm{BV} / \mathrm{TV}$ & $23.9 \pm 3.6$ & $17.7 \pm 3.8$ & $19.6 \pm 4.1$ & $9.8 \pm 1.7$ \\
\hline TbTh, mm & $0.032 \pm 0.003$ & $0.029 \pm 0.002$ & $0.030 \pm 0.002$ & $0.026 \pm 0.001$ \\
\hline \multicolumn{5}{|l|}{ SS mutant } \\
\hline BV/TV & $52.9 \pm 5.7$ & $45.3 \pm 9.5$ & $24.3 \pm 5.1$ & $29.0 \pm 1.8$ \\
\hline TbTh, mm & $0.064 \pm 0.007$ & $0.056 \pm 0.011$ & $0.029 \pm 0.003$ & $0.037 \pm 0.001$ \\
\hline $\mathrm{TbN}, 1 / \mathrm{mm}$ & $8.308 \pm 0.342$ & $7.973 \pm 0.839$ & $5.805 \pm 0.874$ & $7.874 \pm 0.199$ \\
\hline TbTh, mm & $0.038 \pm 0.001$ & $0.054 \pm 0.009^{*}$ & $0.039 \pm 0.003$ & $0.039 \pm 0.003$ \\
\hline $\mathrm{TbN}, 1 / \mathrm{mm}$ & $8.891 \pm 0.509$ & $8.862 \pm 0.459$ & $8.095 \pm 0.576$ & $8.157 \pm 0.687$ \\
\hline TbSp, mm & $0.076 \pm 0.004$ & $0.059 \pm 0.009$ & $0.088 \pm 0.0130$ & $0.088 \pm 0.014$ \\
\hline
\end{tabular}

Values represent the mean \pm standard error ( $n \geq 3$ per group).

${ }^{*} P<0.05$.

mediated phosphorylation of STAT3, made mice obese comparable to the $\Delta 65$ mice, however, the bone loss and marrow adiposity was not as great as observed in the $\Delta 65$ mice and no changes in growth were observed. In addition, the changes were more evident in the male SS mutant male mice compared with female mice. This suggests that the active signaling occurring in the SS is sufficient for growth and can maintain some normal bone phenotype in males and most of the normal phenotype in females. By contrast, the LL mutant mice did not gain weight and in the case of the female mice we observed reduced body weights. Male LL mutant mice were the only

TABLE 2 Vetebral trabecular bone parameters

\begin{tabular}{|c|c|c|c|c|}
\hline & \multicolumn{2}{|l|}{ Male } & \multicolumn{2}{|l|}{ Female } \\
\hline & Control & Mutant & Control & Mutant \\
\hline \multicolumn{5}{|l|}{$\Delta 65$} \\
\hline $\mathrm{BV} / \mathrm{TV}$ & $35.5 \pm 4.0$ & $57.9 \pm 8.7^{*}$ & $40.5 \pm 2.3$ & $54.7 \pm 5.5$ \\
\hline TbTh, mm & $0.039 \pm 0.002$ & $0.058 \pm 0.016$ & $0.044 \pm 0.002$ & $0.059 \pm 0.008$ \\
\hline $\mathrm{TbN}, 1 / \mathrm{mm}$ & $8.923 \pm 0.360$ & $10.145 \pm 1.272$ & $9.069 \pm 0.215$ & $9.319 \pm 0.312$ \\
\hline $\mathrm{TbSp}, \mathrm{mm}$ & $0.073 \pm 0.007$ & $0.041 \pm 0.004^{*}$ & $0.066 \pm 0.004$ & $0.049 \pm 0.006^{*}$ \\
\hline \multicolumn{5}{|l|}{ SS mutant } \\
\hline $\mathrm{BV} / \mathrm{TV}$ & $47.3 \pm 5.7$ & $54.5 \pm 1.9$ & $39.4 \pm 3.5$ & $43.4 \pm 3.5$ \\
\hline TbTh, mm & $0.048 \pm 0.004$ & $0.055 \pm 0.002$ & $0.044 \pm 0.003$ & $0.044 \pm 0.003$ \\
\hline $\mathrm{TbN}, 1 / \mathrm{mm}$ & $9.570 \pm 0.414$ & $9.944 \pm 0.089$ & $8,834 \pm 0.290$ & $9.706 \pm 0.341$ \\
\hline TbSp, mm & $0.056 \pm 0.008$ & $0.046 \pm 0.002$ & $0.070 \pm 0.006$ & $0.059 \pm 0.005$ \\
\hline \multicolumn{5}{|l|}{ LL mutant } \\
\hline $\mathrm{BV} / \mathrm{TV}$ & $39.6 \pm 5.1$ & $53.1 \pm 4.6$ & $44.8 \pm 1.6$ & $35.2 \pm 2.3^{*}$ \\
\hline TbTh, mm & $0.040 \pm 0.003$ & $0.051 \pm 0.002^{*}$ & $0.048 \pm 0.001$ & $0.036 \pm 0.002^{*}$ \\
\hline $\mathrm{TbN}, 1 / \mathrm{mm}$ & $9.780 \pm 0.433$ & $10.287 \pm 0.522$ & $9.190 \pm 0.257$ & $9.658 \pm 0.400$ \\
\hline $\mathrm{TbSp}, \mathrm{mm}$ & $0.064 \pm 0.007$ & $0.048 \pm 0.007$ & $0.061 \pm 0.003$ & $0.069 \pm 0.005$ \\
\hline
\end{tabular}

Bone volume fraction (bone volume/total volume, BV/TV), trabecular thickness (TbTh), trabecular number (TbN), trabecular spacing (TbSp). Values represent the mean \pm standard error $(n \geq 3$ per group).

$* P<0.05$. 

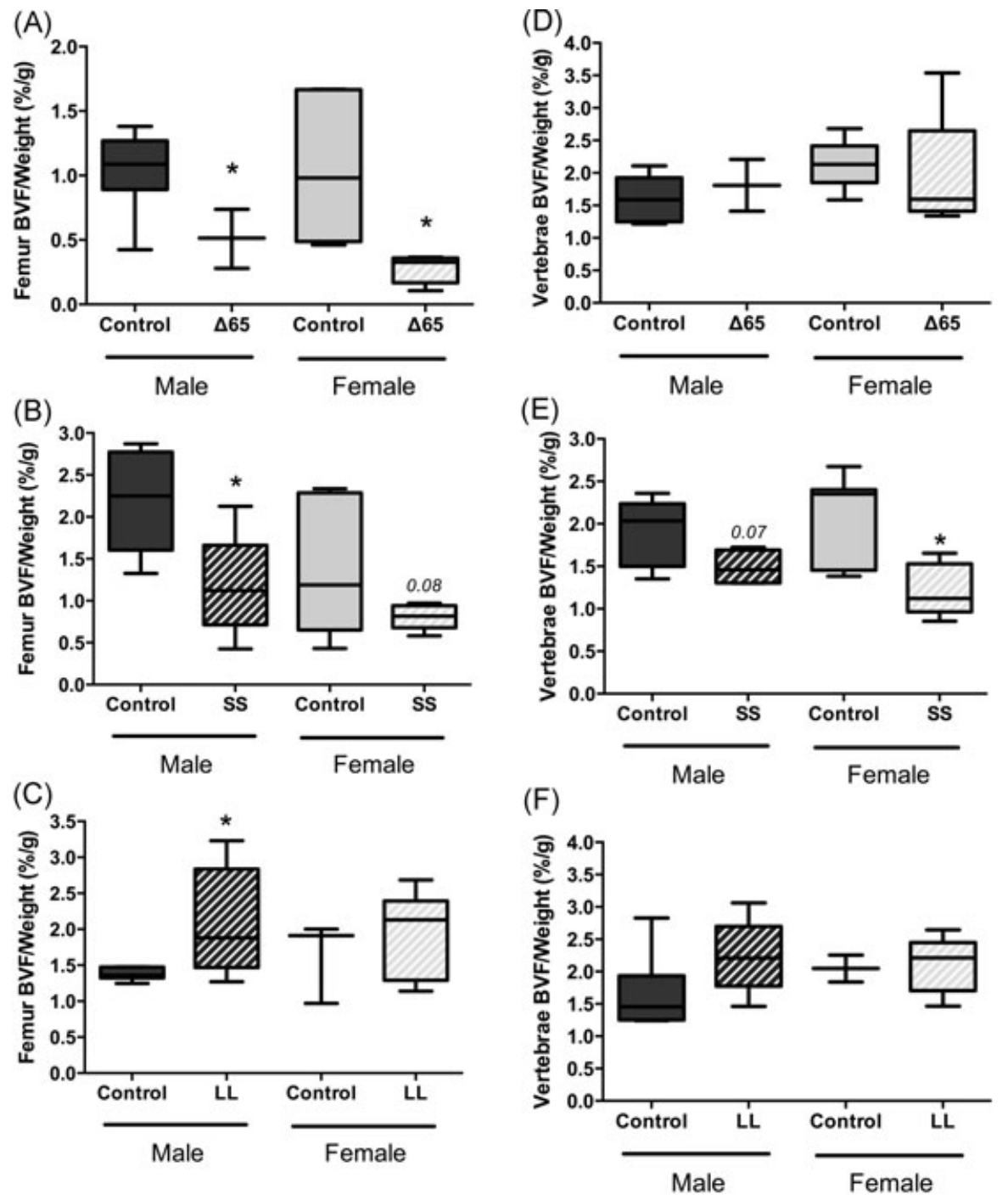

FI G URE 3 LepRb mutations have sex and location-dependent effects on bone volume fraction. Femur (A-C) and vertebral (D-E) bone volume fraction (BVF) was determined, in 8 to 9-week-old mice, by microcomputed tomography and expressed relative to mouse body weight. LepRb mutant mouse data $(\Delta 65, \mathrm{SS}$, or LL) are grouped with data obtained from corresponding littermate control mice. Values represent averages \pm SE. $\Delta 65$ mice, $n \geq 3$ per group; SS and LL mice, $n=6$ to 7 per group; $* P<0.05$

group to show an increase in bone density, which is consistent with leptin hypersensitivity.

Interestingly, when corrected to body weight the $\Delta 65$ mutant mice did not experience vertebral bone loss. This is similar to previous reports showing significant bone loss in femur or tibia, with only mild changes in vertebrae, in $\mathrm{db} / \mathrm{db}$ and ob/ob mice. ${ }^{19,31}$ This is consistent with leptin having site-specific effects on bone. ${ }^{27}$ Unexpectedly, we observed a moderate decrease in $\mathrm{BVF} /$ body weight in the SS mutant vertebrae. This suggests that a signaling pathway affected by the SS mutation, that is, STAT3, may contribute to leptin's location-dependent effects in femur but not vertebrae; thus, pathway inhibition allows a response in vertebrae. Future mechanistic studies are needed to better understand the underlying site-specific differences in the regulation of bone.

Analyses of cortical bone indicated that only the $\Delta 65$ mutation had an impact on male cortical bone parameters but not density. Whereas, the $\Delta 65$ and SS mutation affected female cortical bone density but only the SS mutant had significant negative effects on cortical bone parameters. The LL mutation did not have a major impact on cortical bone in either sex, but females did display a significant decrease in inner cortical perimeter. Thus, the LepRb mutations reveal a sex-dependent response. The $\Delta 65$ mutation affecting male cortical bone, while the SS (significant) and LL (trend) mutants affect cortical bone only in female mice and in opposite ways. This sex-dependent LepRb regulation of cortical parameters needs to be further dissected in the future larger study that identifies the downstream pathways that have opposing influences on cortical bone.

Leptin is a potent regulator of bone marrow adiposity. Increased LepRb signaling can decrease marrow adiposity, ${ }^{10-12,14,33}$ whereas reduced leptin signaling promotes bone marrow adiposity. ${ }^{1,17}$ The latter is most evident in $\mathrm{ob} / \mathrm{ob}$ and $\mathrm{db} / \mathrm{db} .^{34,35}$ In our study, both the $\Delta 65$ mutant and SS mutants had increased femoral bone marrow 

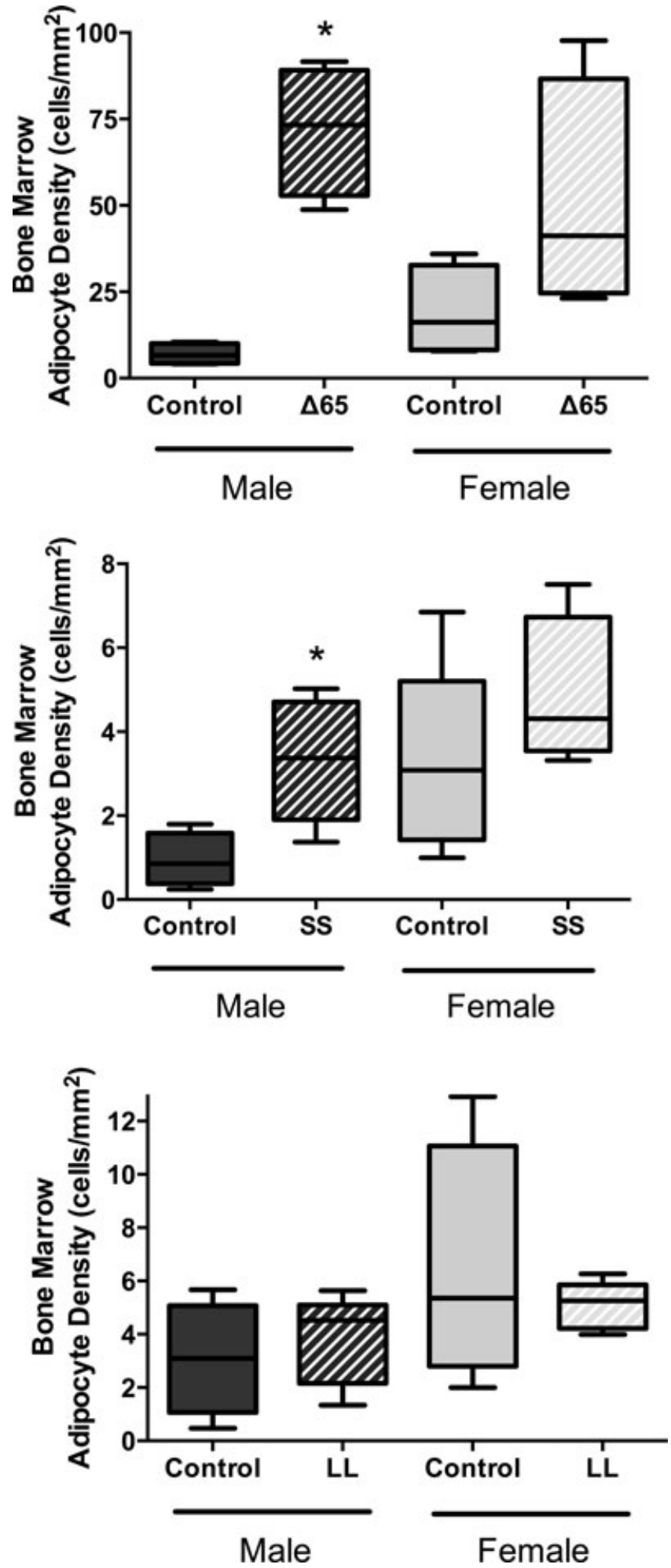

F I G U RE 4 LepRb mutations have sex and location-dependent effects on bone marrow adipocyte density. Distal femur bone marrow, proximal to the growth plate, was examined for marrow adipocytes. Adipocytes were counted and expressed relative to the marrow area. LepRb mutant mouse data $(\Delta 65$, SS, or LL) are grouped with data obtained from corresponding littermate control mice. Values represent averages \pm SE. $n \geq 4$ for all conditions; ${ }^{*} P<0.05$ adiposity. This was more prominent in males than females. In addition, the magnitude of the increase was much greater in $\Delta 65$ mutants than in SS, which lack only $\mathrm{Tyr}_{1138} /$ STAT3 signaling. The LL mutation had no effect on marrow adiposity. Mechanistically, LepRb signaling can regulate adiposity through its expression and signaling in mesenchymal stromal cells and on $94 \%$ of bone marrow-derived colonies that mature to make bone, cartilage, and adipocytes. ${ }^{13}$ In vitro studies demonstrate that leptin promotes bone marrow stromal cell osteogenesis rather than adipogenesis. ${ }^{10-12,14}$ Leptin treatment has been demonstrated to reduce marrow adiposity and corrects skeletal abnormalities in ob/ob mice, though minimal changes in bone occur in rodents capable of producing leptin. ${ }^{2,33}$ Leptin treatment also prevents T1diabetic-induced bone-marrow adiposity, but in this model, leptin treatment is unable to prevent T1-diabetic bone loss. ${ }^{32}$

Leptin has previously been shown to regulate bone growth, in part by its ability to affect chondrocytes. ${ }^{17,28,36}$ Leptin-deficient ob/ob mice have fragile growth plates with disturbed columnar structures and increased apoptosis, a phenotype abolished by treatment with leptin. ${ }^{28}$ Interestingly, only the $\Delta 65$ mutation, lacking all tyrosine kinase signaling from LepRb, causes reduced bone length. Neither the LL or SS mutant mice had an observable growth defect in the femur, suggesting that full inhibition of LepRb-mediated tyrosine kinase signaling is required to obtain notable growth stunting. The lack of a growth effect in LL mice is consistent with a previous study that found no difference in snout-anus length in LL mutant versus WT mice. ${ }^{22}$ In our study, we also found no change in vertebral growth as determined by vertebrae height measures. This is consistent with many of the leptin effects being targeted to long bones rather than axial bones. ${ }^{27}$

It should be noted that in contrast to total body LepRb signaling modulation, as in ob/ob, $\mathrm{db} / \mathrm{db}$ and our studies, the targeted deletion of LepRb in bone marrow mesenchymal stromal cells causes a different phenotype characterized by increased osteogenesis and decreased adipogenesis and increased fracture healing. ${ }^{11,37}$ These studies point out the complexity of LepRb signaling in the body, which encompasses effects on the brain, immune system, metabolism, and eating behavior, ${ }^{8,9,16,18,20}$ all of which impact bone. Our study suggests that the modulation of whole body LepRb signaling may outweigh the contribution of MSC LepRb regulation of bone and thus lead to increased marrow adiposity and decreased bone density. While it is critical to understand the role of LepRb signaling within the bone as well as in other individual tissues such as brain, our studies provide important 
TABLE 3 Cortical bone parameters

\begin{tabular}{|c|c|c|c|c|}
\hline & \multicolumn{2}{|l|}{ Male } & \multicolumn{2}{|l|}{ Female } \\
\hline & Control & Mutant & Control & Mutant \\
\hline \multicolumn{5}{|l|}{$\Delta 65$} \\
\hline Mean thickness, mm & $0.180 \pm 0.008$ & $0.145 \pm 0.003^{* *}$ & $0.174 \pm 0.004$ & $0.169 \pm 0.006$ \\
\hline Inner, $\mathrm{mm}$ & $3.764 \pm 0.047$ & $4.071 \pm 0.195$ & $3.759 \pm 0.052$ & $3.529 \pm 0.181$ \\
\hline Outer, mm & $4.868 \pm 0.076$ & $4.934 \pm 0.230$ & $4.774 \pm 0.065$ & $4.533 \pm 0.199$ \\
\hline Marrow area, $\mathrm{mm}^{2}$ & $0.995 \pm 0.022$ & $1.034 \pm 0.035$ & $0.994 \pm 0.032$ & $0.906 \pm 0.056$ \\
\hline Cortical area, $\mathrm{mm}^{2}$ & $0.687 \pm 0.041$ & $0.468 \pm 0.028^{* *}$ & $0.588 \pm 0.032$ & $0.545 \pm 0.042$ \\
\hline $\mathrm{BMD}, \mathrm{mg} / \mathrm{cc}$ & $937 \pm 10$ & $885 \pm 10$ & $983 \pm 14$ & $922 \pm 19^{* *}$ \\
\hline \multicolumn{5}{|l|}{ SS mutant } \\
\hline Mean thickness, mm & $0.245 \pm 0.008$ & $0.255 \pm 0.010$ & $0.200 \pm 0.007$ & $0.203 \pm 0.007$ \\
\hline Inner, $\mathrm{mm}$ & $3.720 \pm 0.082$ & $3.619 \pm 0.109$ & $3.497 \pm 0.056$ & $3.775 \pm 0.089^{*}$ \\
\hline Outer, mm & $5.246 \pm 0.091$ & $5.232 \pm 0.132$ & $4.733 \pm 0.055$ & $5.007 \pm 0.038^{*}$ \\
\hline Marrow area, $\mathrm{mm}^{2}$ & $0.985 \pm 0.043$ & $0.942 \pm 0.055$ & $0.870 \pm 0.025$ & $0.989 \pm 0.041^{*}$ \\
\hline Cortical area, $\mathrm{mm}^{2}$ & $1.041 \pm 0.039$ & $1.072 \pm 0.060$ & $0.752 \pm 0.040$ & $0.772 \pm 0.041$ \\
\hline BMD, mg/cc & $1076 \pm 15$ & $1081 \pm 16$ & $1023 \pm 12$ & $955 \pm 18^{* *}$ \\
\hline \multicolumn{5}{|l|}{$L L$ mutant } \\
\hline Mean thickness, mm & $0.211 \pm 0.007$ & $0.219 \pm 0.006$ & $0.205 \pm 0.007$ & $0.201 \pm 0.009$ \\
\hline Inner, mm & $3.726 \pm 0.042$ & $3.780 \pm 0.068$ & $3.686 \pm 0.070$ & $3.513 \pm 0.039 *$ \\
\hline Outer, mm & $5.029 \pm 0.030$ & $5.126 \pm 0.067$ & $4.948 \pm 0.091$ & $4.761 \pm 0.072$ \\
\hline Marrow area, $\mathrm{mm}^{2}$ & $0.983 \pm 0.021$ & $1.001 \pm 0.032$ & $0.942 \pm 0.026$ & $0.895 \pm 0.022$ \\
\hline Cortical area, $\mathrm{mm}^{2}$ & $0.853 \pm 0.033$ & $0.902 \pm 0.038$ & $0.780 \pm 0.054$ & $0.779 \pm 0.052$ \\
\hline $\mathrm{BMD}, \mathrm{mg} / \mathrm{cc}$ & $979 \pm 28$ & $1026 \pm 26$ & $999 \pm 19$ & $994 \pm 26$ \\
\hline
\end{tabular}

Abbreviation: BMC, bone mineral density.

Values represent the mean \pm standard error ( $n \geq 3$ per group).

${ }^{*} P<0.05$.

${ }^{* *} P<0.01$ compared with corresponding wild-type control.

TABLE 4 Summary of findings

\begin{tabular}{|c|c|c|c|c|c|c|c|c|c|c|c|c|}
\hline \multirow[b]{2}{*}{ Lepr mutants: } & \multicolumn{2}{|c|}{$\begin{array}{l}\text { Body } \\
\text { weight }\end{array}$} & \multicolumn{2}{|c|}{$\begin{array}{l}\text { Femur } \\
\text { BVF/wt }\end{array}$} & \multicolumn{2}{|c|}{$\begin{array}{l}\text { Marrow } \\
\text { adiposity }\end{array}$} & \multicolumn{2}{|c|}{$\begin{array}{l}\text { Vertebrae } \\
\text { BVF/wt }\end{array}$} & \multicolumn{2}{|c|}{$\begin{array}{l}\text { Cortical } \\
\text { parameters }\end{array}$} & \multicolumn{2}{|c|}{ Bone growth } \\
\hline & Male & Female & Male & Female & Male & Female & Male & Female & Male & Female & Male & Female \\
\hline$\Delta 65$ & $\uparrow$ & $\uparrow$ & $\downarrow$ & $\downarrow$ & $\uparrow \uparrow \uparrow$ & $\uparrow \uparrow$ & - & - & $\Delta$ & $\Delta$ & $\downarrow$ & $\downarrow$ \\
\hline SS & $\uparrow$ & $\uparrow$ & $\downarrow$ & $\vdots$ & $\uparrow$ & $\hat{i}$ & $\vdots$ & $\downarrow$ & - & $\Delta$ & - & - \\
\hline $\mathbf{L L}$ & - & $\downarrow$ & $\uparrow$ & - & - & - & - & - & - & - & - & - \\
\hline
\end{tabular}

Abbreviations: $\uparrow$, significant increase; $\downarrow$ significant decrease; $\uparrow$, trend; $\Delta$, change; BVF, bone volume fraction.

Changes are relative to littermate sex and age-matched controls.

insight into potential systemic effects of leptin, which contribute bone density regulation.

\section{5 | CONCLUSIONS}

Similar to leptin-receptor deficient $\mathrm{db} / \mathrm{db}$ mice, truncation of the signaling domain of LepRb in the $\Delta 65$ mutant led to increased body mass, decreased femoral trabecular bone volume, bone length, and cortical thickness, and increased bone marrow adiposity (Table 4). The SS mutant, which lacks $\mathrm{Tyr}_{1138} / \mathrm{STAT} 3$ signaling, recapitulated the excess body mass phenotype of the $\mathrm{db} / \mathrm{db}$ and $\Delta 65$ mice. However, its impact on bone parameters was reduced. Specifically, femoral length and cortical thickness were normal and marrow adiposity, though marginally increased, was an order of magnitude less than $\Delta 65$ animals. This suggests that signals in addition to $\mathrm{Tyr}_{1138} / \mathrm{STAT} 3$ are necessary to 
promote maximal bone loss, growth restriction, and marrow fat accumulation. In the absence of excess peripheral and bone marrow adiposity, as present in LL mice, bone mass was unchanged (females), or increased (males). These results are relevant to understanding how disruptions in leptin signaling, whether due to monogenetic or diet-induced obesity, may impact bone development and growth. This may be particularly relevant in the context of juvenile obesity, where loss of leptin signaling via LepRb could compromise bone development and growth. Understanding how leptin modifies bone through LepRb will impact the design of strategies to promote bone growth and integrity in such at-risk populations.

\section{ACKNOWLEDGEMENTS}

The authors thank the Investigative Histology Laboratory in the Department of Physiology, Division of Human Pathology for their assistance with bone histology as well as the Biomedical Imaging Center for use of the microcomputed tomography imaging. They also thank to Daniel Barnett for his technical expertize and Drs Fraser Collins and Natalie Wee for their critical review of the manuscript. These studies were supported by funding from the National Institute of Health (grants numbers RO1 DK101050, RO1 AT007695, R01 DK056731, and R00 DE024178).

\section{CONFLICTS OF INTEREST}

The authors declare that there are no conflicts of interest.

\section{ORCID}

\section{Laura R. McCabe (D) http://orcid.org/0000-0001-} 5407-162X

\section{REFERENCES}

1. Hamrick MW. Leptin, bone mass, and the thrifty phenotype. J Bone Miner Res. 2004;19(10):1607-1611.

2. Iwaniec UT, Boghossian S, Lapke PD, Turner RT, Kalra SP. Central leptin gene therapy corrects skeletal abnormalities in leptin-deficient ob/ob mice. Peptides. 2007;28(5):1012-1019.

3. Iwaniec UT, Boghossian $\mathrm{S}$, Trevisiol $\mathrm{CH}$, Wronski TJ, Turner RT, Kalra SP. Hypothalamic leptin gene therapy prevents weight gain without long-term detrimental effects on bone in growing and skeletally mature female rats. $J$ Bone Miner Res. 2011;26(7):1506-1516.

4. Karsenty G. Convergence between bone and energy homeostases: leptin regulation of bone mass. Cell Metab. 2006; 4(5):341-348.

5. Motyl KJ, Rosen CJ. Understanding leptin-dependent regulation of skeletal homeostasis. Biochimie. 2012;94(10):2089-2096.
6. Reid IR. Leptin deficiency-lessons in regional differences in the regulation of bone mass. Bone. 2004;34(3):369-371.

7. Elefteriou F, Ahn JD, Takeda S, et al. Leptin regulation of bone resorption by the sympathetic nervous system and CART. Nature. 2005;434(7032):514-520.

8. Abella V, Scotece M, Conde J, et al. Leptin in the interplay of inflammation, metabolism and immune system disorders. Nat Rev Rheumatol. 2017;13(2):100-109.

9. Villanueva EC, Myers MG, Jr. Leptin receptor signaling and the regulation of mammalian physiology. Int J Obes. 2008;32(suppl 7):S8-S12.

10. Reseland JE, Syversen U, Bakke I, et al. Leptin is expressed in and secreted from primary cultures of human osteoblasts and promotes bone mineralization. J Bone Miner Res. 2001;16 (8):1426-1433.

11. Scheller EL, Song J, Dishowitz MI, Soki FN, Hankenson KD, Krebsbach PH. Leptin functions peripherally to regulate differentiation of mesenchymal progenitor cells. Stem Cells. 2010;28(6):1071-1080.

12. Thomas T, Gori F, Khosla S, Jensen MD, Burguera B, Riggs BL. Leptin acts on human marrow stromal cells to enhance differentiation to osteoblasts and to inhibit differentiation to adipocytes. Endocrinology. 1999;140(4):1630-1638.

13. Zhou BO, Yue R, Murphy MM, Peyer JG, Morrison SJ. Leptinreceptor-expressing mesenchymal stromal cells represent the main source of bone formed by adult bone marrow. Cell Stem Cell. 2014;15(2):154-168.

14. Cornish J, Callon KE, Bava U, et al. Leptin directly regulates bone cell function in vitro and reduces bone fragility in vivo. $J$ Endocrinol. 2002;175(2):405-415.

15. Hamrick MW, Della Fera MA, Choi YH, Hartzell D, Pennington C, Baile CA. Injections of leptin into rat ventromedial hypothalamus increase adipocyte apoptosis in peripheral fat and in bone marrow. Cell Tissue Res. 2007;327(1):133-141.

16. Philbrick KA, Martin SA, Colagiovanni AR, Branscum AJ, Turner RT, Iwaniec UT. Effects of hypothalamic leptin gene therapy on osteopetrosis in leptin-deficient mice. J Endocrinol. 2018;236(2):57-68.

17. Steppan CM, Crawford DT, Chidsey-Frink KL, Ke H, Swick AG. Leptin is a potent stimulator of bone growth in ob/ob mice. Regul Pept. 2000;92(1-3):73-78.

18. Turner RT, Kalra SP, Wong CP, et al. Peripheral leptin regulates bone formation. J Bone Miner Res. 2013;28(1):22-34.

19. Williams GA, Callon KE, Watson M, et al. Skeletal phenotype of the leptin receptor-deficient $\mathrm{db} / \mathrm{db}$ mouse. J Bone Miner Res. 2011;26(8):1698-1709.

20. Lindenmaier LB, Philbrick KA, Branscum AJ, Kalra SP, Turner RT, Iwaniec UT. Hypothalamic leptin gene therapy reduces bone marrow adiposity in ob/ob mice fed regular and high-fat diets. Front Endocrinol. 2016;7:110.

21. Gong Y, Ishida-Takahashi R, Villanueva EC, Fingar DC, Münzberg H, Myers MG, Jr. The long form of the leptin receptor regulates STAT5 and ribosomal protein S6 via alternate mechanisms. J Biol Chem. 2007;282(42):3101931027.

22. Björnholm M, Münzberg H, Leshan RL, et al. Mice lacking inhibitory leptin receptor signals are lean with normal endocrine function. J Clin Invest. 2007;117(5):1354-1360. 
23. Banks AS, Davis SM, Bates SH, Myers MG, Jr. Activation of downstream signals by the long form of the leptin receptor. J Biol Chem. 2000;275(19):14563-14572.

24. Münzberg H, Huo L, Nillni EA, Hollenberg AN, Bjørbæk C. Role of signal transducer and activator of transcription 3 in regulation of hypothalamic proopiomelanocortin gene expression by leptin. Endocrinology. 2003;144(5):2121-2131.

25. Bates SH, Stearns WH, Dundon TA, et al. STAT3 signalling is required for leptin regulation of energy balance but not reproduction. Nature. 2003;421(6925):856-859.

26. Robertson S, Ishida-Takahashi R, Tawara I, et al. Insufficiency of Janus kinase 2-autonomous leptin receptor signals for most physiologic leptin actions. Diabetes. 2010;59(4):782-790.

27. Hamrick MW, Pennington C, Newton D, Xie D, Isales C. Leptin deficiency produces contrasting phenotypes in bones of the limb and spine. Bone. 2004;34(3):376-383.

28. Kishida Y, Hirao M, Tamai N, et al. Leptin regulates chondrocyte differentiation and matrix maturation during endochondral ossification. Bone. 2005;37(5):607-621.

29. He H, Liu R, Desta T, Leone C, Gerstenfeld LC, Graves DT. Diabetes causes decreased osteoclastogenesis, reduced bone formation, and enhanced apoptosis of osteoblastic cells in bacteria stimulated bone loss. Endocrinology. 2004;145(1): 447-452.

30. Ramos-Junior ES, Leite GA, Carmo-Silva CC, et al. Adipokine chemerin bridges metabolic dyslipidemia and alveolar bone loss in mice. J Bone Miner Res. 2017;32(5):974-984.

31. Ealey KN, Fonseca D, Archer MC, Ward WE. Bone abnormalities in adolescent leptin-deficient mice. Regul Pept. 2006;136 (1-3):9-13.
32. Motyl KJ, McCabe LR. Leptin treatment prevents type I diabetic marrow adiposity but not bone loss in mice. $J$ Cell Physiol. 2009;218(2):376-384.

33. Hamrick MW, Della-Fera MA, Choi Y-H, Pennington C, Hartzell D, Baile CA. Leptin treatment induces loss of bone marrow adipocytes and increases bone formation in leptindeficient ob/ob mice. J Bone Miner Res. 2005;20(6):994-1001.

34. Devlin MJ, Cloutier AM, Thomas NA, et al. Caloric restriction leads to high marrow adiposity and low bone mass in growing mice. J Bone Miner Res. 2010;25(9):2078-2088.

35. Ecklund K, Vajapeyam S, Feldman HA, et al. Bone marrow changes in adolescent girls with anorexia nervosa. J Bone Miner Res. 2010;25(2):298-304.

36. Gat-Yablonski G, Phillip M. Leptin and regulation of linear growth. Curr Opin Clin Nutr Metab Care. 2008;11 (3):303-308.

37. Yue R, Zhou BO, Shimada IS, Zhao Z, Morrison SJ. Leptin receptor promotes adipogenesis and reduces osteogenesis by regulating mesenchymal stromal cells in adult bone marrow. Cell Stem Cell. 2016;18(6):782-796.

How to cite this article: McCabe IC, Fedorko A, Myers MG Jr, Leinninger G, Scheller E, McCabe LR. Novel leptin receptor signaling mutants identify location and sex-dependent modulation of bone density, adiposity, and growth. $J$ Cell Biochem. 2019;120:4398-4408. https://doi.org/10.1002/jcb.27726 